or above the umbilicus. Free fluid in the peritoneal cavity is very unusual. As a rule fluctuation in the tumour is obscure, and there is a doughy feeling on palpation. Above all, irregular degrees of consistence in different portions are very characteristic of ovarian dermoids. When quite small the cyst not rarely lies altogether in the pelvis, and occasionally remains there even after attaining a considerable size, in which case it is a distinct source of danger if the patient becomes pregnant.

Complications often occur early. Ovarian dermoids are particularly liable to torsion of the pedicle, intracystic hæmorrhage, the formation of adhesions due to inflammation of neighbouring peritoneum, septic infection, and escape of the contents from the cyst cavity, e.g., into bladder or rectum, or, if due to rupture, into the peritoneum. Even very early in its history a dermoid may be tender to palpation, a fact which is an aid to diagnosis. A sudden attack of acute pain is suggestive of a twisted pedicle. The existence of a dermoid tumour in a pregnant woman is a matter of gravity. As a rule gestation is not prejudiced, but complications are liable to arise as pregnancy advances, and during delivery, while the cyst is much exposed to infection during the puerperium. Experience has shown that the removal of a dermoid during pregnancy is not dangerous, whilst abortion does not necessarily follow the procedure, and should it ensue it hardly interferes with recovery from the operation. Hence a pregnant patient who has an ovarian cyst should be strongly advised to have the tumour removed without delay.

1 Medical Press and Circular, Feb. 10th, 1904.

\section{THE EARLY DIAGNOSIS OF CONSUMPTION.}

IN the light of accumulated experience in the modern treatment of pulmonary tuberculosis it has become a matter of supreme importance to recognise the condition at the earliest possible moment. The success or failure of treatment will depend largely upon this point-whether the patient be treated in a sanatorium or not. As Dr. Cattle ${ }^{1}$ remarks, sanatorium treatment is no panacea ; even incipient and seemingly favourable cases sometimes become worse in spite of every effort. Yet, speaking generally, the earliest cases are those which do the best. Dr. Cattle deprecates the modern tendency towards leaving the diagnosis to bacteriologists. He points out that although the presence of tubercle bacilli in the sputum is a certain proof of the presence of tuberculosis in the lungs or some part of the respiratory passages, yet in early cases, where evidence is most wanted, bacilli often cannot be discovered, and their absence from the sputum cannot be held to negative a diagnosis of pulmonary tuberculosis. It is well, therefore, not to waste valuable time in repeated attempts to obtain a positive bacteriological result, in the presence of appreciable physical signs of the disease.

In considering the physical signs it must be borne in mind that slight differences between the apices of the two lungs are frequently to be met with even in quite healthy individuals. At the normal right spex there may be slight dulness on percussion, more audible breath - sounds, and more obvious vocal fremitus and vocal resonance. These differences are due to the fact that the right bronchus is wider, shorter, and more horizontal than the left, while it enters the lung at a higher level and gives off the bronchus to the upper lobe nearer the trachea than that for the left upper lobe is given off. For these reasons the glottic sounds are better conducted to the right apex. These peculiarities may cause error in two directions; either slightly more audible, but normal, sounds at the right apex may be regarded as pathological, or commencing signs of consolidation may be regarded as being within the physiological limit. As the right apex is more frequently affected with tuberculosis than the left, the difficulty in discriminating between health and disease is enhanced. If, on the other hand, there is slight] dulness at the left apex, if the breath-sounds, voca resonance and fremitus equal or exceed in intensity those on the right, there is almost certainly disease. Error is liable in left-handed patients, for in them the left apex is commonly less resonant than the right.

In addition to examining the sputum for bacilli and carefully investigating the physical signs another means of diagnosis which is occasionally of use is the injection of Koch's tuberculin. The patient's history and present general state of health must also be duly considered, and it must be remembered that true hæmoptysis in the great majority of instances, even in the absence of physical signs, signifies tuberculosis, though it is necessary to exclude the possibility of the blood coming from a lesion of the nose, mouth, pharynx, larynx, or stomach.

1 Practitioner, February 1904.

\section{THE AFTER-HISTORY OF GASTRIC ULCER.}

Dr. JAMEs Russeli ${ }^{1}$ has collected the histories of 47 cases of gastric ulcer after the patients had left the Birmingham General Hospital, where they were treated during the years 1892-1899. All his cases were in women who had suffered from hæmatemesis, and the length of each case was counted from the first hæmatemesis. One patient or $2 \cdot 1$ per cent. died directly from her gastric disease. Two died from intercurrent maladies. Thirteen or $27 \cdot 7$ per cent. had recovered for periods from two to eight years, without relapse. Seven cases (14 9 per cent) recovered after relapses. These relapses had occurred at periods varying from three or four months to seven years after the first bleeding. In only two of these had hæmatemesis recurred. Four cases are difficult to classify, because in one a gastric operation was performed and in the other three the evidence of recovery is unsatisfactory. Twenty - one patients $(44 \cdot 7$ per cent.) still suffer from their complaint, the duration being from two to 13 years. Nine or 10 of these had a second hæmorrhage, and in two cases a third is recorded. Recurrent hæmatemesis while not precluding the possibility of recovery is a serious factor in prognosis pointing at least to a considerable prolongation of the period of active disease. Regarding these cases of non-recovery, Dr. Russell believes that it would be unwise to attempt to draw any definite conclusion as to the number of 